\title{
Evolutionary Stability Analysis of the Coal Mine Safety Management System Governed by Delay and Impulsive Differential Equations
}

\author{
Li Bai, ${ }^{1}$ Xinhua Wang $\mathbb{D}^{1},{ }^{1}$ Rongwu Lu $\mathbb{D}^{1,2}$ Hao Yu $\mathbb{D}^{1},{ }^{1}$ and Dan Li $\mathbb{D}^{1,2}$ \\ ${ }^{1}$ College of Economics and Management, Shandong University of Science and Technology, Qingdao 266590, China \\ ${ }^{2}$ College of Mathematics and Systems Science, Shandong University of Science and Technology, Qingdao 266590, China
}

Correspondence should be addressed to Rongwu Lu; lurong5@sdust.edu.cn and Hao Yu; yh2001v20@163.com

Received 16 February 2020; Revised 15 April 2020; Accepted 20 April 2020; Published 28 May 2020

Academic Editor: Chittaranjan Hens

Copyright (c) $2020 \mathrm{Li}$ Bai et al. This is an open access article distributed under the Creative Commons Attribution License, which permits unrestricted use, distribution, and reproduction in any medium, provided the original work is properly cited.

Control of unsafe behaviors has always been a difficult point in Coal Mine Safety Management (CMSM). To study the regular pattern of behavior propagations in CMSM, a series of evolutionary dynamical system models are established by the delay and impulsive differential equations (DIDEs). The threshold constraints (TCs) and impulse responses in the models can accurately correspond to the actual inspection and supervision measures in safety management. Through the simulation analysis and comparison of the system stability, it can be concluded that some proper settings of the TCs and impulse responses bring satisfactory evolutionary stability. Based on the main results of this study, we put forward some decision-making suggestions on the control points and control strategies of unsafe behaviors in safety management.

\section{Introduction}

Coal mine safety responsibility accidents are the results of the continuous accumulation of unsafe behaviors, and the spread of unsafe behaviors is the main cause of the accumulation of unsafe behaviors $[1,2]$. But the occurrence of coal mine safety accidents is uncertain at any moment, and employees have differences in risk perception and preferences, cognitive abilities, etc. Therefore, although some employees know that unsafe behaviors (illegal operations) may cause safety accidents, they might still choose unsafe behavior and become the implementer of unsafe behaviors under the influence of such factors as production tasks, extra payoffs, and trust to luck. China's coal mine production is mostly arranged in district groups. There are a large amount of contacts between employees, which can communicate and transmit various information and then affect each other's behaviors. The spread of behavior is not limited to the field of social bad behavior, but also spreads in other fields of behavior. Many scholars have also used virus propagation models or system dynamics theories and methods to study the laws of behavioral propagation from different aspects [3-5].
The policies of safety management in nowadays China are always adjusted with the occurrence of accidents. The relevant supervision measures are characterized by accident types and lack of comprehensive analysis and cannot take certain effects in the long run. Actually, CMSM utilizes a combination of economic, institutional, and cultural methods to control unsafe behaviors in coal mining. Its goal is to restrain the occurrence frequencies of the unsafe behaviors and improve the coal mine safety. As a controlling method of unsafe behaviors, CMSM has already attracted many scholars' attention [6-10]. Based on the theoretical analysis of the evolutionary stability strategy, we have already put forward some incentive measures and restraint measures to control the instability of player's dynamical behaviors in CMSM [11]. It is found that game strategy selection is controllable under the corresponding measures, and appropriate incentive and constraint measures can mobilize the initiative of safe behaviors. However, those flexible incentive and restraint measures for the excess subsidies and severe punishments may bring some unexpected economic or social problems, which make some small and medium coal mine enterprises unable to implement those measures [12-14]. 
The delay and impulsive differential equations (DIDEs) have been widely used in biological control, artificial intelligence, and other fields [15-22]. There are a large number of systems in various engineering fields that continuously change in a certain time interval. Due to some artificial intervention behaviors, the system state will be disturbed for a relatively short period of time, which will cause the system state to change instantly. This phenomenon is also common in CMSM. It actually takes a certain amount of time delays to check the safe behaviors and feedbacks on the inspection results, and safety management measures would be altered much more strictly when colliery safety entered a tough situation (such as a safety accident just happened in a coal mine and many other coal mines have similar production conditions with it), which corresponds exactly to the impulse response in the models. The dynamical system governed by the DIDEs is one of the right methods to analyze those sudden changes in the system state [23-27]. So in this paper, we want to set up some evolutionary game models with time delay and state feedback impulse to analyze the behavioral communications of those participants in CMSM. This microperspective analysis method can make an in-depth analysis of unsafe behavior's communications, and we believe that the design of control mechanisms for unsafe behaviors is the key to promote the long-term effectiveness of safety measures in CMSM.

The rest of this paper is organized as follows. In Section 2, we build an evolutionary game model in CMSM by the DIDEs with threshold constraint (TC) on $x_{1}$. Then, in Section 3, we carry out detailed and in-depth stability analysis of the DIDEs with TC on $x_{1}$, which involves various possible pulse states. In Section 4, we carry out stability analysis of the DIDEs with TCs on $x_{3}$ or $x_{2}$. In Section 5, this paper is finally concluded and some suggestions are put forward to improve the evolutionary stability in CMSM.

\section{Preliminaries}

There are many factors that affect the game behaviors of each interested group [28]. If the employees are completely rational, the behavior selection process can be described by the expected utility function theory. But in reality the employees are not completely rational, and the behavior selection is mainly based on the psychological expectation and perceived value of the profit and loss value of the behavior choice. Behavioral communication between employees often occurs in every coal mine process. CMSM also involves many interest parties and there are complex relationships between them. Generally, there are at least three participants involved: coal miners (denoted as player I), safety inspection groups inside the coal mine (player II), and safety regulatory departments of local government (player III). In evolutionary game theory, the advantage of a population is reflected in the proportion of participants. A multiparty evolutionary game model has been established to analyze the selection strategies of safety behaviors in our previous work, and we have obtained the following replicator dynamics [29]:

$$
\left\{\begin{array}{l}
\dot{x}_{1}=x_{1}\left(1-x_{1}\right)\left[\left(x_{2}+\left(1-x_{2}\right) x_{3}\right) f_{1}-c_{1}\right], \\
\dot{x}_{2}=x_{2}\left(1-x_{2}\right)\left[\left(1-x_{1}\right)\left(f_{1}+x_{3} f_{2}\right)-c_{2}\right], \\
\dot{x}_{3}=x_{3}\left(1-x_{3}\right)\left[\left(1-x_{1}\right)\left(1-x_{2}\right)\left(f_{1}+f_{2}\right)-c_{3}\right],
\end{array}\right.
$$

where $x_{1}, x_{2}$, and $x_{3}$ are the probabilities of those players choosing safety behaviors (safe operations, or safe inspections, or safe supervisions), respectively. $c_{i}(i=1,2,3)$ are, respectively, the safety costs (inputs) paid by player I, player II, and player III, and $f_{i}(i=1,2)$ are the penalty index when player I and player II make mistakes (unsafe behaviors) and are checked out, respectively. For the value of the parameter, we make an explanation here in particular. The values of the parameters are relative, i.e., the values of the parameters represent the costs and punishments paid by the participants for the benefit of a unit.

According to the actual situation of China's coal mine production and coal mine safety regulation, these parameters are assumed to meet the following conditions:

$$
\begin{aligned}
& \text { (i) } c_{2} \leq c_{3} \leq c_{1} \leq 1 \\
& \text { (ii) } f_{i} \geq 1, i=1,2
\end{aligned}
$$

When the implementation of the employees' unsafe behaviors can bring them additional benefits, they may be imitated and learned by other employees so that the unsafe behaviors can be spread among the employees. Many operating activities in coal mine production are completed through the mutual cooperation of group members. Group members are regulated and restricted by group norms, thereby maintaining roughly uniform behavioral goals and cognitive structures. Some unsafe behaviors have a group nature, such as violations of regulation production. This kind of group unsafe behavior sometimes happens under the influence of higher-level illegal command, group norms, and other factors [30-33].

As for the situation of CMSM, we can make further consideration on the participants' group unsafe behaviors. If one conducts safe/unsafe behaviors in coal mine production, it always takes a period of time (inspection and supervision come one after another) to receive the consequences (wages, subsidies, or penalties). The characteristic of the delay differential equations is that the right end of the equation does not depend on the current moment, but depends on the function values of the past moment. Hence, we consider the following DIDEs:

$$
\left\{\begin{array}{l}
\dot{x}_{1}(t)=x_{1}\left(t-\tau_{1}\right)\left(1-x_{1}\left(t-\tau_{1}\right)\right)\left[\left(x_{2}\left(t-\tau_{1}\right)+x_{3}\left(t-\tau_{3}\right)-x_{2}\left(t-\tau_{2}\right) x_{3}\left(t-\tau_{3}\right)\right) f_{1}-c_{1}\right], \\
\dot{x}_{2}(t)=x_{2}\left(t-\tau_{2}\right)\left(1-x_{2}\left(t-\tau_{2}\right)\right)\left[\left(1-x_{1}\left(t-\tau_{1}\right)\right)\left(f_{1}+x_{3}\left(t-\tau_{3}\right) f_{2}\right)-c_{2}\right], \\
\dot{x}_{3}(t)=x_{3}\left(t-\tau_{3}\right)\left(1-x_{3}\left(t-\tau_{3}\right)\right)\left[\left(1-x_{1}\left(t-\tau_{1}\right)\right)\left(1-x_{2}\left(t-\tau_{2}\right)\right)\left(f_{1}+f_{2}\right)-c_{3}\right], \\
\Delta x_{2}(t)=\alpha, \alpha>0 \\
\Delta x_{3}(t)=\beta, \beta>0
\end{array}\right\} x_{1} \leq x_{1}^{*},
$$


where $\tau_{1}, \tau_{2}$, and $\tau_{3}$ are, respectively, the time delays for $x_{1}, x_{2}$, and $x_{3}$, which denote the times between players receiving payoffs and their behaviors been done. Because player I receives inspections and supervision from the other two players and even colleagues in the same shift, player I knows the consequences in the fastest time. Similarly, the frequency of player II's safety inspections is faster than that of player III. So, it is assumed that

$$
0<\tau_{1} \leq \tau_{2} \leq \tau_{3} .
$$

Coal mine workers are the most direct producers of unsafe behaviors, and they are also the ultimate managed parties in safety management. If $x_{1}$ is found to be less than a threshold $x_{1}^{*}$ (threshold is a certain number, which exists in some mature colliery enterprises or coal industry associations) after every fixed time $T$ (pulse period, which is the time interval between two adjacent pulses in a periodic repeating pulse sequence), such impulses will be generated as $\Delta x_{2}(t)$ for $x_{2}$ and $\Delta x_{3}(t)$ for $x_{3}$, respectively. Therefore, the DIDEs (2) denote an evolutionary dynamical system with TC on $x_{1}$ in CMSM.

The exact solution of the evolutionary dynamical system (2) is undoubtedly difficult to obtain, so the model analysis process is mainly carried out through simulation analysis. The general process of numerical simulations is described briefly as follows:

Step 1: the entire time period is divided into $N$ intervals of the same length $t$ span, and let $i=1$, the initial values of $\left(x_{1}, x_{2}, x_{3}\right)$ are given $\left.\left(x_{1}, x_{2}, x_{3}\right)\right|_{t=0}$.

Step 2: in ith interval, the delay differential equations (DDEs) with constant delays (i.e., the first three equations in the DIDEs) are solved by MATLAB Function dde23 with given time delays and initial values of $\left.\left(x_{1}, x_{2}, x_{3}\right)\right|_{t=i-1}$, and some figures such as phase diagram and TSG can be drawn by plot3/plot function of MATLAB. Then, at the right end of the interval, the solutions are checked whether the pulse trigger condition (TC) is met. If TC is not satisfied, the value of the solution vector at the last moment is the initial value when DIDEs are solved in the next interval. Otherwise, the pulse value is added to the solution vector at the last moment to obtain the initial value of the next interval.

Step 3: $i=i+1$, loop to step 2 until the DIDEs are solved and simulated in the entire time period.

In the simulation process in this section, the model parameters are set as follows: $c_{1}=0.5 ; c_{2}=0.2$; $c_{3}=0.3 ; f_{1}=1 ; f_{2}=1.2 ; \alpha=0.3 ; \beta=0.2$. In order to illustrate the generality and consistency of this study, we first analyze the game dynamic system without time delay and impulse. Let $\tau_{1}=\tau_{2}=\tau_{3}=0$ and $x_{1}^{*}=1$ in DIDEs (2). Figures 1(a) and 1(b) are time series graphs (TSGs) of players' strategy selection probabilities $\left(x_{1}, x_{2}, x_{3}\right)$ when the dynamical system (2) has no pulse and no delay. The initial values of $\left(x_{1}, x_{2}, x_{3}\right)$ are $P_{0}(0.5,0.3,0.2)$ and $P_{1}(0.7,0.5,0.3)$, respectively.

As can be seen in Figure 1, there are repeated periodic fluctuations in the game process no matter what the initial value is. It illustrates that participants in the safety management system constantly change their choices based on the coal mine safety status and the behavioral selections of others. When player I performs well ( $x_{1}$ takes a large value), player II will relax its supervision ( $x_{2}$ decreases). Then, the value of $x_{1}$ will gradually decrease, and then, $x_{2}$ will increase, and then, $x_{1}$ will increase again. The dynamical game system enters a cyclical process, where player III plays a smaller and smaller role ( $x_{3}$ gradually decreases towards zero). Once coal mine safety enters such a periodic fluctuation state, the efficiency of safety production management can hardly be guaranteed.

Due to the sequential nature of production behaviors and supervision behaviors, the variables $x_{1}, x_{2}$, and $x_{3}$ certainly have time delays. However, the impulse response is usually artificially set, which means the supervisory behaviors suddenly change or become stronger in reality. So we should take pulse responses and TCs into account and perform detailed stability analysis on the dynamical system to demonstrate the influence of the setting of pulses and TCs.

\section{Stability Analysis on the DIDEs with TC on $x_{1}$ in CMSM}

In this section, to reflect the correspondence and influence of time delays and pulses in the dynamical system (2), the evolutionary stability of CMSM is studied and simulated in a variety of cases. In the general theoretical study of the stability of dynamic systems, the stability of each variable needs to meet certain requirements. Since this study takes safety management as the application background and the main object of behavior control is player I, the stability analysis in this article is mainly on $x_{1}$. The behavior control strategies of player II and player III are regarded as the adjustments of safety management decisions, and there are no special requirements for the stability of $x_{2}$ or $x_{3}$.

During model simulation, lags $=\left(\tau_{1}, \tau_{2}, \tau_{3}\right)$ denotes the vector of the delay values of variables $x_{i}, i=1,2,3$, which have been previously mentioned in Section 2. From now on, we set lags $=(3,5,7) / 30$ in every process of the model simulations in this study, which are a bit small. It is based on the consideration that behavioral communication between employees in coal mining enterprises is fast and significant, where mostly a large number of workers are engaged in the same workflow and workplace, which nearly unchanged for a long time. Besides, the value of pulse period $T=7$, which never changes but in Section 4.3, where we will point out specially.

3.1. The DIDEs (2) Have Time Delays but No Pulse. As shown in Figure 2, there are similar fluctuations with Figure 1 when the dynamical system (2) has time delays but no pulse. Although the fluctuation period of the variable $x_{1}$ is gradually increasing, the fluctuation of $x_{2}$ is becoming more severe. There is also no stability in safety management in this case, and the effectiveness of safety management will not be satisfactory. 
TSG of the DDEs with no pulse and no delay,

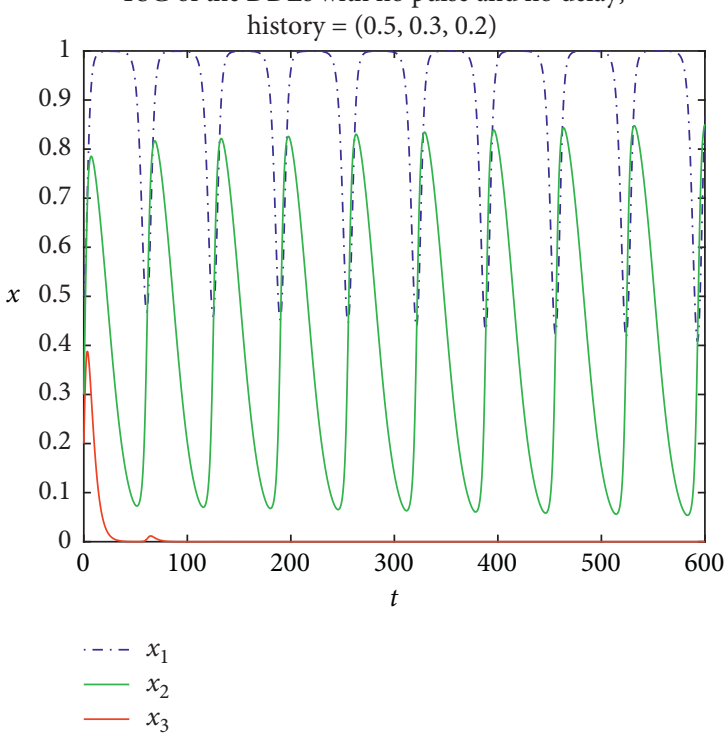

(a)

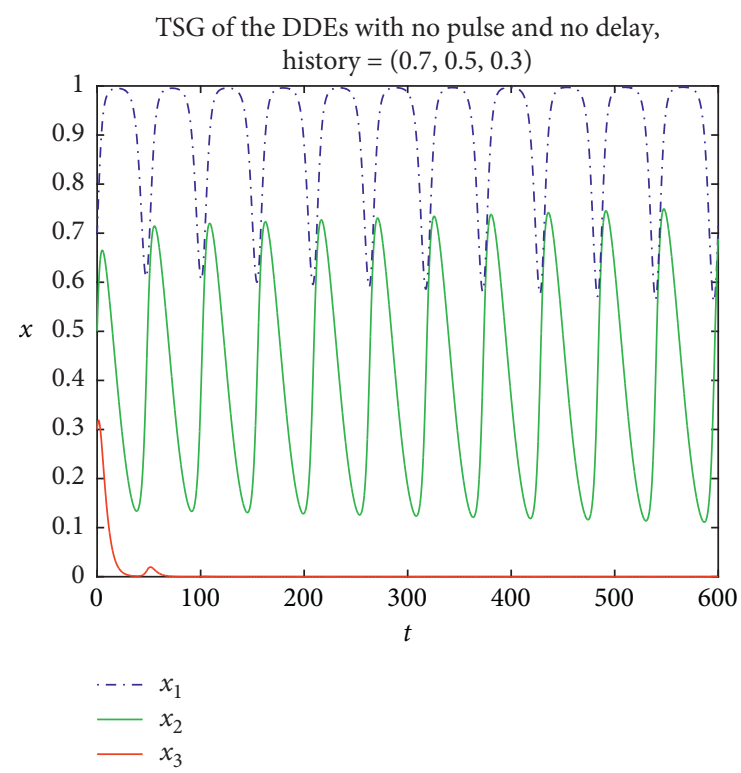

(b)

FIGURE 1: TSGs of $x_{i}$ when the DIDEs (2) have no pulse and no delay. (a) $\left.\left(x_{1}, x_{2}, x_{3}\right)\right|_{t=0}=P_{0}(0.5,0.3,0.2)$ and (b) $\left.\left(x_{1}, x_{2}, x_{3}\right)\right|_{t=0}=P_{1}(0.7,0.5,0.3)$.



(a)



(b)

Figure 2: TSGs when the DIDEs (2) have delays but no pulse. Lags $=(3,5,7) / 30$. (a) $\left.\left(x_{1}, x_{2}, x_{3}\right)\right|_{t=0}=P_{0}(0.5,0.3,0.2)$ and (b) $\left.\left(x_{1}, x_{2}, x_{3}\right)\right|_{t=0}=P_{1}(0.7,0.5,0.3)$.

3.2. The DIDEs (2) Have Pulses. The implementers of the safety supervision measures are player II and player III, while player I is the object of its application. The pulse setting for player I has no practical meaning in CMSM. Therefore, we should not design any pulse for $x_{1}$, but apply a fixed value pulse to $x_{2}$ or $x_{3}$. As mentioned earlier in Section 2, a pulse on $x_{2}$ or $x_{3}$ corresponds to the strengthening or adjustment of safety supervision measures. Specifically, if $x_{3}$ has a pulse, it can correspond to strengthen the safety supervision of government authorities (such as the increase in the frequency of safety inspections, or the expansion of its scope). When $x_{2}$ has a pulse, the safety inspection team of coal mine enterprises will strengthen their supervision level in proportion. As the dynamical system is found not very dependent on variables' 



(a)


(b)

Figure 3: TSGs of DIDEs (2) when $x_{3}$ has a pulse $(\beta=0.2) .\left.\left(x_{1}, x_{2}, x_{3}\right)\right|_{t=0}=P_{1}(0.7,0.5,0.3)$. (a) $x_{1}^{*}=0.5$ and (b) $x_{1}^{*}=0.6$.

initial values from Figures 1 and 2, the initial value of $\left(x_{1}, x_{2}, x_{3}\right)$ is given as $P_{1}(0.7,0.5,0.3)$ in this section.

Figure 3 shows TSGs of DIDEs (2) when $x_{3}$ has a pulse separately $(\alpha=0 ; \beta=0.2)$, where all the three variables $x_{1}, x_{2}$, and $x_{3}$ involve in a cyclical fluctuation process. It illustrates that $x_{3}$ becomes smaller and is even close to zero after a period of time, and then, the pulse takes its effect. In this case, player III actively participates in the safety supervision. As $x_{1}^{*}$ changes its value from 0.5 to 0.6 , the peaks of $x_{2}$ and $x_{3}$ are relatively reduced, but the fluctuation of $x_{1}$ is more frequent.

Figure 4 shows TSGs of DIDEs (2) when $x_{2}$ has a pulse separately $(\alpha=0.3 ; \beta=0)$. It shows $x_{1}$ still has no stability, and $x_{2}$ fluctuates more violently while $x_{3}$ gradually decreases to zero. As $x_{1}^{*}$ changes its value from 0.5 to 0.6 , the fluctuation of $x_{1}$ finally weakens while the peak of $x_{2}$ intensifies. However, the system stability does not improve significantly.

Figure 5 shows TSGs of DIDEs (2) when both $x_{2}$ and $x_{3}$ have pulses $(\alpha=0.3 ; \beta=0.2)$. We can find that $x_{1}=1$ lasts even longer and $x_{2}$ and $x_{3}$ are more actively involved in the dynamical system. And, the dynamical system (2) reduces volatility when $x_{1}^{*}$ increases. However, all of these three cases shown in Figures 3-5 are still oscillating waveforms and there is no essential change from the previous case shown in Figure 2. It can be concluded from the simulation results in this section that there is almost no evolutionary stability in the dynamical system with TC on $x_{1}$ (i.e., the DIDEs (2)).

Therefore, the probability of player I's safety behavior selection should not be taken as a prerequisite condition for adopting safety supervision measures in the practice of safety management. Although coal miners are the ultimate object and the basic part for the implementation of safety management in CMSM, safety management of coal miners is the most difficult part due to the large number and the largescale differences of skill and knowledge. Therefore, we analyze the evolutionary stability of the dynamical system where there are TCs imposed on other variables.

\section{Stability Analysis on the Dynamical System with TCs on $x_{2}$ or $x_{3}$}

Compared with the coal miners, staff members in safety inspection groups of the coal mine enterprise, or in the safety supervision departments of the government, have higher knowledge and technical level and safety quality but less quantity. Their organizational integrity is much stronger, and the adjustment of their safety decisions should be more effective and faster. So in this section, we conduct stability analysis on the dynamical system when the TCs are imposed on $x_{2}$ or $x_{3}$.

4.1. Stability Analysis on the DIDEs with TC on $x_{3}$. The safety supervision departments of the government (player III in the dynamical systems) play a leading role in the entire process of coal mine safety supervision [34-36]. As policy makers and direct implementers in CMSM, they are responsible for organizing, supervising, and supporting the relevant units in coal mine enterprises to perform the duties of safety inspections and timely coordinating and solving major problems in coal mine safety. The policy adjustment of the government safety departments undoubtedly can make an important impact in CMSM. Hence, we consider the following DIDEs with a TC on $x_{3}$ : 

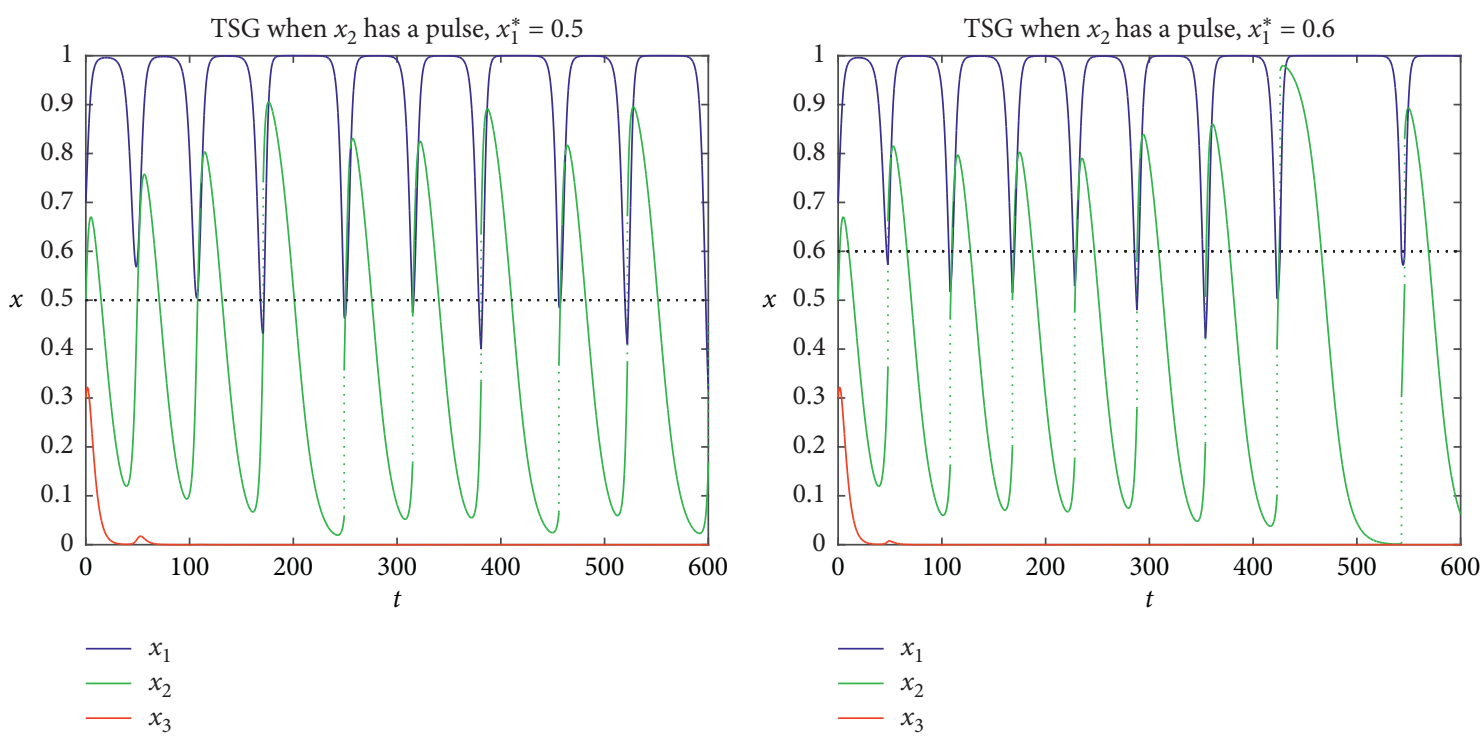

(a)

(b)

Figure 4: TSGs of DIDEs (2) when $x_{2}$ has a pulse $(\alpha=0.3) .\left.\left(x_{1}, x_{2}, x_{3}\right)\right|_{t=0}=P_{1}(0.7,0.5,0.3)$. (a) $x_{1}^{*}=0.5$ and $(\mathrm{b}) x_{1}^{*}=0.6$.


(a)

(b)

FIgURE 5: TSGs of DIDEs (2) when both $x_{2}$ and $x_{3}$ have pulses $(\alpha=0.3 ; \beta=0.2) .\left.\left(x_{1}, x_{2}, x_{3}\right)\right|_{t=0}=P_{1}(0.7,0.5,0.3)$. (a) $x_{1}^{*}=0.5$ and (b) $x_{1}^{*}=0.6$.

$$
\left\{\begin{array}{l}
\dot{x}_{1}(t)=x_{1}\left(t-\tau_{1}\right)\left(1-x_{1}\left(t-\tau_{1}\right)\right)\left[\left(x_{2}\left(t-\tau_{1}\right)+x_{3}\left(t-\tau_{3}\right)-x_{2}\left(t-\tau_{2}\right) x_{3}\left(t-\tau_{3}\right)\right) f_{1}-c_{1}\right], \\
\dot{x}_{2}(t)=x_{2}\left(t-\tau_{2}\right)\left(1-x_{2}\left(t-\tau_{2}\right)\right)\left[\left(1-x_{1}\left(t-\tau_{1}\right)\right)\left(f_{1}+x_{3}\left(t-\tau_{3}\right) f_{2}\right)-c_{2}\right], \\
\dot{x}_{3}(t)=x_{3}\left(t-\tau_{3}\right)\left(1-x_{3}\left(t-\tau_{3}\right)\right)\left[\left(1-x_{1}\left(t-\tau_{1}\right)\right)\left(1-x_{2}\left(t-\tau_{2}\right)\right)\left(f_{1}+f_{2}\right)-c_{3}\right], \\
\Delta x_{2}(t)=\alpha, \alpha \geq 0 \\
\Delta x_{3}(t)=\beta, \beta \geq 0
\end{array}\right\} x_{3} \leq x_{3}^{*} .
$$



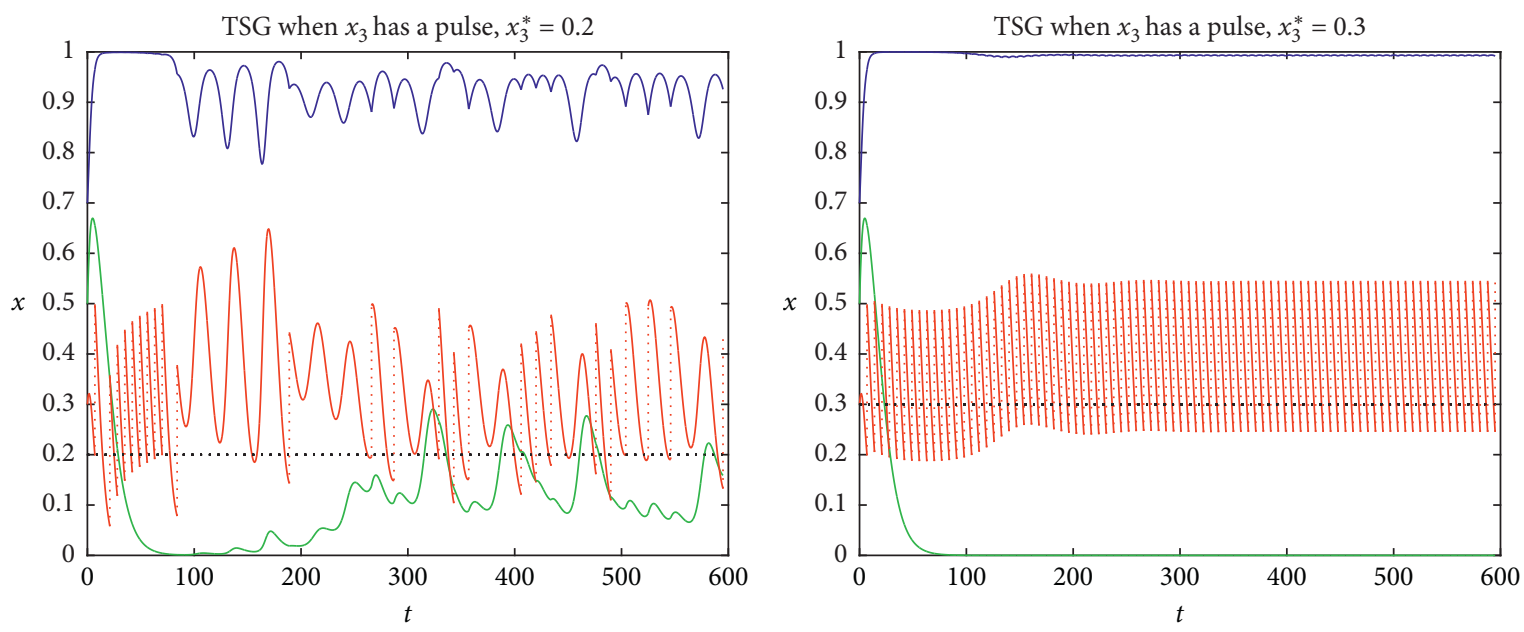



(a)
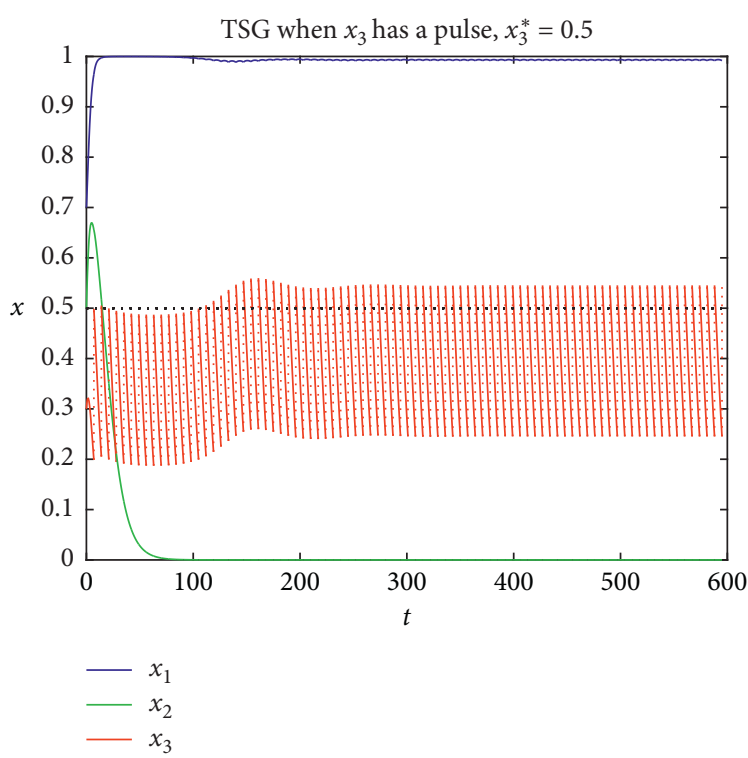

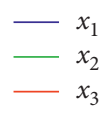

(b)


(c)

(d)

Figure 6: TSGs of DIDEs (4) with TC on $x_{3}$ when $x_{3}$ has a pulse $(\alpha=0.3) .\left.\left(x_{1}, x_{2}, x_{3}\right)\right|_{t=0}=P_{1}(0.7,0.5,0.3) .(\mathrm{a}) x_{3}^{*}=0.2$, (b) $x_{3}^{*}=0.3,(\mathrm{c})$ $x_{3}^{*}=0.5$, and $(\mathrm{d}) x_{3}^{*}=0.6$.

To analyze the influence of the safety supervision departments adjusting their behaviors on the evolutionary stability of dynamic system (4), we consider just one case here, i.e., $x_{3}$ individually imposed a pulse $(\beta)$.

As can be seen from Figure 6, the dynamical system (4) has a satisfactory evolutionary stability when $x_{3}^{*}$ is large enough. When $x_{3}^{*}$ is not less than $0.3, x_{1}$ will steadily approaching 1. Even when $x_{3}^{*}=0.2$, which is a bit small value, the volatility of the dynamical system becomes very weak and $x_{1}$ gradually stabilizes to a relatively large value (see Figure 6(a)). So it is concluded that the adjustment of government departments' safety measures plays an important role in improving the safety management level of coal mine.

4.2. Stability Analysis on the DIDEs (4) with TC on $x_{2}$. In accordance with the previous section, the effect of the decision adjustment of player II (safety inspection groups inside coal mine enterprises) on CMSM is studied in this section. When there is a threshold constraint (TC) on $x_{2}$, we get the following DIDEs: 





(a)

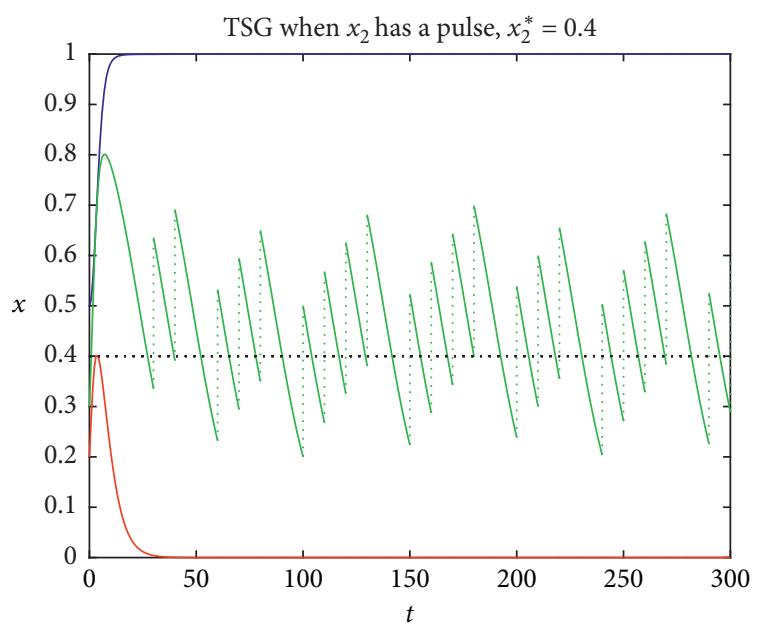

$$
\begin{array}{r}
-x_{1} \\
-x_{2} \\
-x_{3}
\end{array}
$$

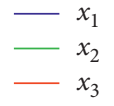

(b)

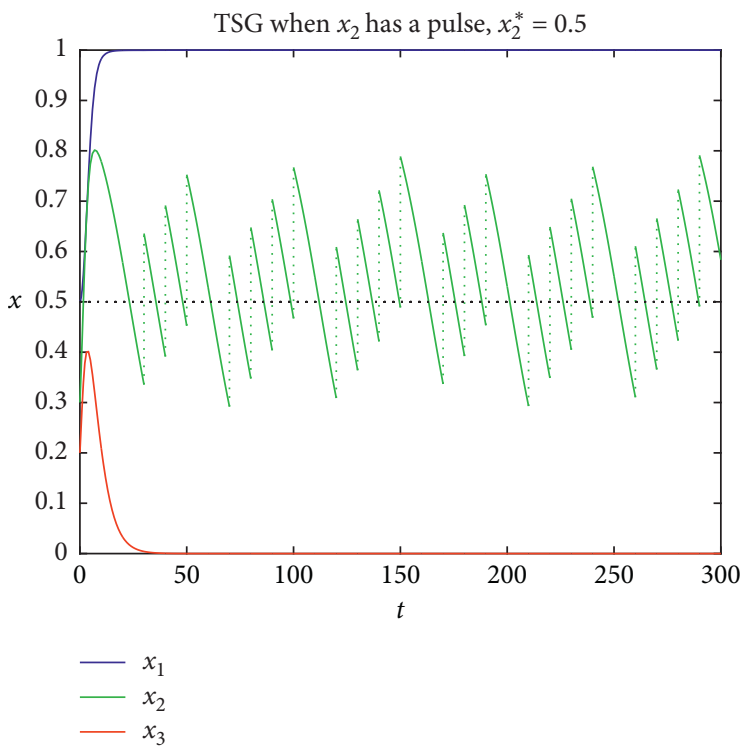

(d)

FIgURe 7: TSGs of $x_{i}$ with TC on $x_{2}$ when $x_{2}$ has a pulse $(\beta=0.2) .\left.\left(x_{1}, x_{2}, x_{3}\right)\right|_{t=0}=P_{0}(0.5,0.3,0.2) .(\mathrm{a}) x_{2}^{*}=0.2$, (b) $x_{2}^{*}=0.3$, (c) $x_{2}^{*}=0.4$, and (d) $x_{2}^{*}=0.5$.

$$
\left\{\begin{array}{l}
\dot{x}_{1}(t)=x_{1}\left(t-\tau_{1}\right)\left(1-x_{1}\left(t-\tau_{1}\right)\right)\left[\left(x_{2}\left(t-\tau_{1}\right)+x_{3}\left(t-\tau_{3}\right)-x_{2}\left(t-\tau_{2}\right) x_{3}\left(t-\tau_{3}\right)\right) f_{1}-c_{1}\right] \\
\dot{x}_{2}(t)=x_{2}\left(t-\tau_{2}\right)\left(1-x_{2}\left(t-\tau_{2}\right)\right)\left[\left(1-x_{1}\left(t-\tau_{1}\right)\right)\left(f_{1}+x_{3}\left(t-\tau_{3}\right) f_{2}\right)-c_{2}\right] \\
\dot{x}_{3}(t)=x_{3}\left(t-\tau_{3}\right)\left(1-x_{3}\left(t-\tau_{3}\right)\right)\left[\left(1-x_{1}\left(t-\tau_{1}\right)\right)\left(1-x_{2}\left(t-\tau_{2}\right)\right)\left(f_{1}+f_{2}\right)-c_{3}\right] \\
\Delta x_{2}(t)=\alpha, \alpha \geq 0 \\
\Delta x_{3}(t)=\beta, \beta \geq 0
\end{array}\right\} x_{2} \leq x_{2}^{*} .
$$

Here, we also consider just one case, i.e., $x_{2}$ individually imposed a pulse ( $\alpha$ ), to analyze the evolutionary stability of the dynamic system (4). Some of the simulation results are shown in Figure 7.
As can be seen from Figure 7, the dynamical system (5) also has a satisfactory evolutionary stability when $x_{2}^{*}$ is large enough (not less than 0.3). Therefore, it is also concluded that the decision adjustment of safety 




(a)

TSG with more TCs and pulses, $x_{1}^{*}=0.9, x_{2}^{*}=0.3$,



(c)
TSG with more TCs and pulses, $x_{1}^{*}=0.8, x_{2}^{*}=0.2$,

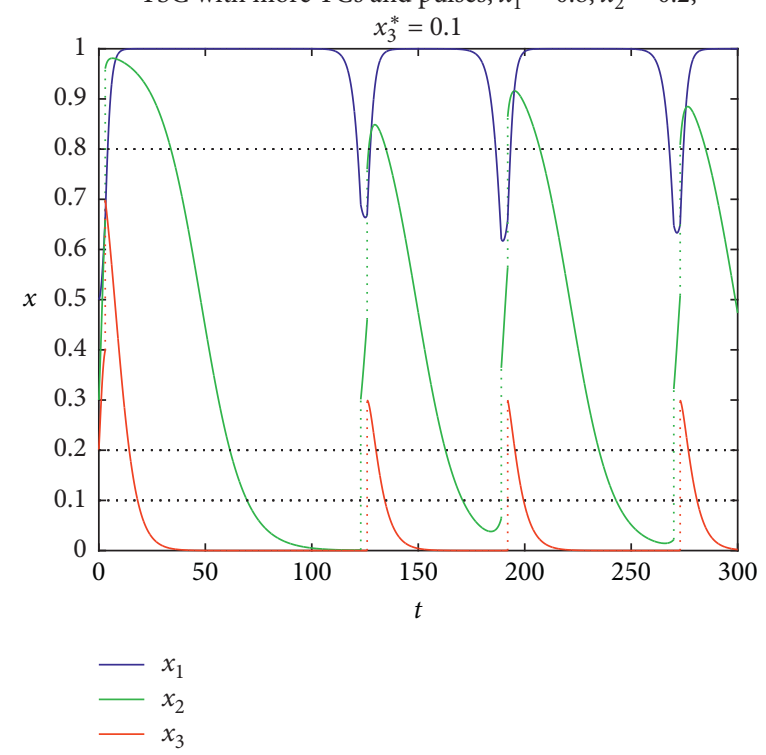

(b)

TSG with more TCs and pulses, $x_{1}^{*}=0.9, x_{2}^{*}=0.2$,

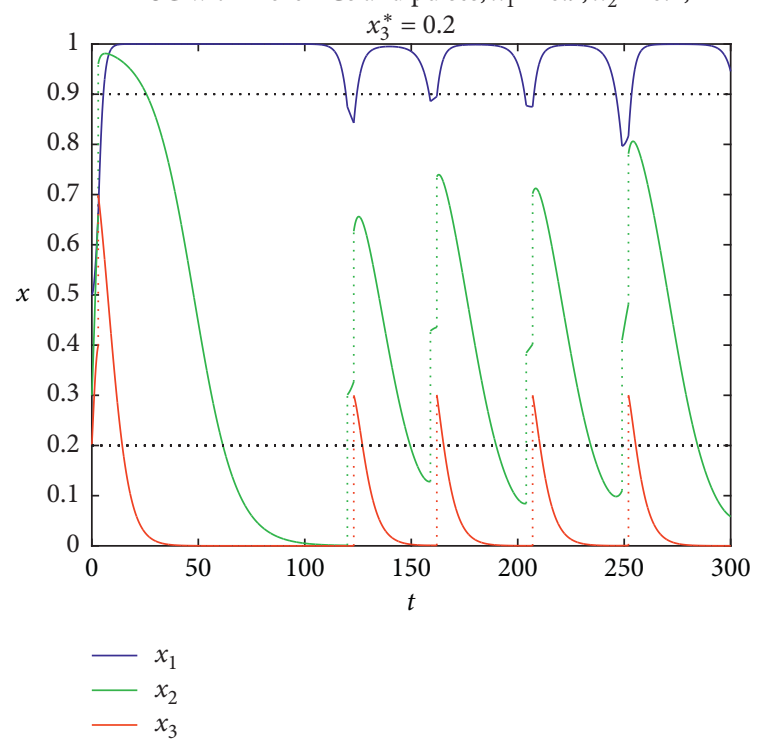

(d)

FIgURE 8: TSGs of the DIDEs (5) with more TCs $(\alpha=0.3, \beta=0.2)$. Pulse period $T=3,\left.\quad\left(x_{1}, x_{2}, x_{3}\right)\right|_{t=0}=P_{0}(0.5,0.3,0.2)$. (a) $x_{1}^{*}=0.6, x_{2}^{*}=0.2, x_{3}^{*}=0.1$, (b) $x_{1}^{*}=0.8, x_{2}^{*}=0.2, x_{3}^{*}=0.1$, (c) $x_{1}^{*}=0.9, x_{2}^{*}=0.3, x_{3}^{*}=0.1$, and (d) $x_{1}^{*}=0.9, x_{2}^{*}=0.2, x_{3}^{*}=0.3$.

TABLE 1: Evolutionary stabilities of the dynamical system with different TCs.

\begin{tabular}{|c|c|c|c|c|}
\hline TCs & Stability of $x_{1}$ & Performance of $x_{2}$ & Performance of $x_{3}$ & Pictures \\
\hline$x_{3} \leq x_{3}^{*}=0.3$ & Evolutionary stable & Inactive & Active & Figures $6(\mathrm{~b})-6(\mathrm{~d})$ \\
\hline$x_{2} \leq x_{2}^{*}=0.3$ & Evolutionary stable & Active & Inactive & Figures $7(\mathrm{~b})-7(\mathrm{~d})$ \\
\hline More TCs combined & Progressively stable & Active & Active & Figures $8(\mathrm{c})$ and $8(\mathrm{~d})$ \\
\hline
\end{tabular}


inspection groups can promote the safety management level of coal mining.

4.3. Stability Analysis on the Dynamical System with More TCs. We have studied the evolutionary stability of the dynamical system with TC imposed on the variables $x_{1}, x_{2}$, and $x_{3}$, respectively, in Section 3 and Sections 4.1 and 4.2. As a neutralization of the previous cases, we consider the following DIDEs, where there are more TCs in CMSM:

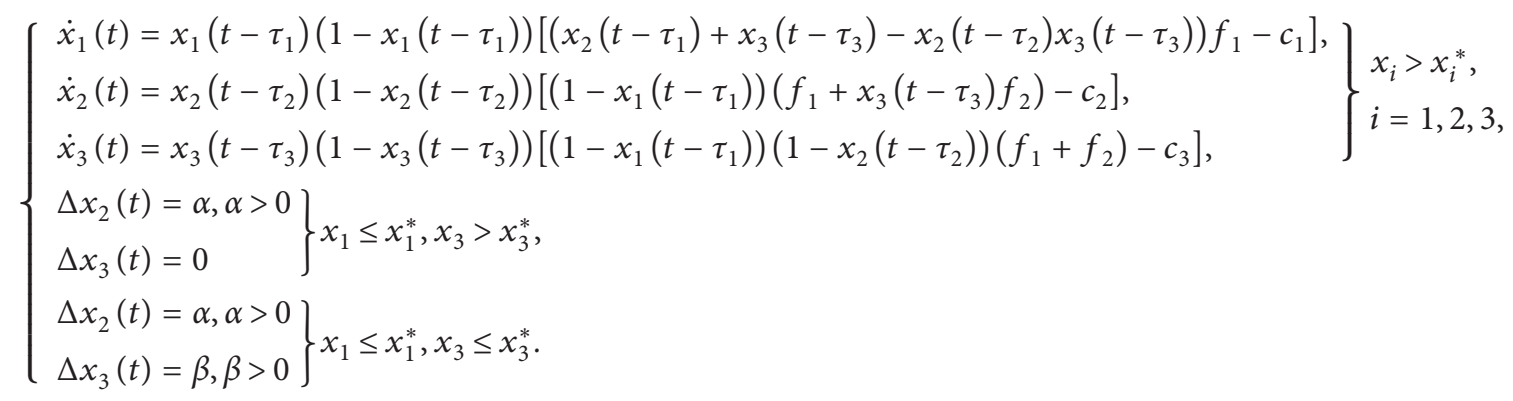

To reflect the impact of TCs, we set pulse period $T=3$ in the model simulations of the DIDES (6), and thus, the pulse check frequency is higher than before. By contrasting Figures $6-8$, the results of the simulation show that the system stability in this case is no better than the previous two cases. The volatility of $x_{1}$ may last forever (see Figure 8).

But on the other hand, these results can also be acceptable because of the relatively large values of $x_{1}$, especially when the level of safety management technology is relatively low, which is still common in some remote areas or smalland medium-sized coal mining enterprises in China [37]. The satisfactory evolutionary stability is difficult to achieve in the practice of CMSM. As shown in Figures 8(c) and 8(d), once the pulse heuristic conditions (the TCs in the DIDEs (6)) are set properly, the dynamic system can make moderate fluctuations over a reasonable range, where the troughs of $x_{1}$ are almost not less than the set value of $x_{1}^{*}$. Meanwhile, all participants in CMSM can restrict and promote each other and actively engage in safety management. That is exactly in line with the aspirations of the Chinese government: to deal with safety management, all participators should take part in the whole process and manage it together.

In this section, stability analyses are made on the dynamical system with different TCs. Table 1 shows the simulation results briefly. When the TCs are imposed on $x_{2}$ or $x_{3}$ separately, $x_{1}$ is evolutionary stable but one of the two supervisors performs inactively. In the last case, when more TCs are combined in the dynamical system, it is gratifying that $x_{1}$ is progressively stable and both the other two have active performances. It is a good revelation for us. We should appropriately adjust the TCs and the thresholds of them, so that the management effect reaches the actual demand of CMSM in practice.

\section{Conclusions}

In this paper, a series of evolutionary dynamical system models governed by the DIDEs are established to study the behaviors communications in CMSM, and stability analysis on the dynamical system with various TCs and pulse responses is carried out. Satisfactory evolutionary stability can be achieved when TCs and pulses are imposed on $x_{2}$ or $x_{3}$, which is very valuable in the practice of CMSM. The TCs and pulses in the models can accurately correspond to the actual inspection and supervision measures in safety management. Therefore, the research conclusions can provide a theoretical basis for the scientific analysis methods for the spread of unsafe behaviors and the formulation of control strategies of the supervisory measures in safety management.

Based on the main results of this article, we attempt to put forward the following suggestions. To improve the management efficiency of coal mine safety, it is not enough to impose various safety regulations on coal miners separately. All the efforts of government departments, safety management departments inside enterprises, and other parties should concentrate to work together. When formulating supervision measures, it is necessary to make targeted arrangements for the safety supervision and inspection commissions of government departments and enterprise's safety inspection groups, so as to highlight the leading role and supervision effectiveness of these safety authorities. Besides, reasonable settings of pulses and TCs can effectively improve the stability of the evolution model and enhance the application value of this research, which require continuous testing in practice of coal mine.

The method of DIDEs is used to carry out the evolutionary stability analysis of behavior propagations in CMSM. It is also the research significance and main innovation of this article, yet there are still some details here for further research and analysis. In future, we can further analyze and study the time unit of the variables of the DIDEs in this study, as well as the appropriate values of pulses, the pulse periods, pulse heuristic conditions (TCs), and impulse response function,. The model research must be closely linked to the practices of specific coal mine enterprises so as to improve the accuracy of the conclusions. In addition, the settings of different model parameters may also cause the 
game dynamic system to enter a chaotic state or have a bifurcation [38-40]. This may cause difficulties in safety management, but it may also be an opportunity to improve the level of safety management.

\section{Data Availability}

The data used to support the findings of this study are included within the article.

\section{Conflicts of Interest}

The authors declare that there are no conflicts of interest regarding the publication of this paper.

\section{Acknowledgments}

This work was supported by the National Natural Science Foundation of China (Grant no. 51574157): Research on Multilateral Game and Control Strategies in Coal Mine Safety System.

\section{References}

[1] R. Liu, W. Cheng, Y. Yu, and Q. Xu, "Human factors analysis of major coal mine accidents in China based on the HFACSCM model and AHP method," International Journal of Industrial Ergonomics, vol. 68, pp. 270-279, 2018.

[2] G. Fu, X. Xie, Q. Jia, W. Tong, and Y. Ge, "Accidents analysis and prevention of coal and gas outburst: understanding human errors in accidents," Process Safety and Environmental Protection, vol. 134, pp. 1-23, 2020.

[3] D. L. Lee, "Social dynamics management and functional behavioral assessment," Journal of Emotional and Behavioral Disorders, vol. 26, no. 1, pp. 62-64, 2018.

[4] L. B. Landon, G. L. Douglas, M. E. Downs et al., "The behavioral biology of teams: multidisciplinary contributions to social dynamics in isolated, confined, and extreme environments," Frontiers in Psychology, vol. 10, 2019.

[5] Nicola Bellomo, A. Bellouquid, L. Gibelli, and N. Outada, "Modeling social behavioral dynamics," in A Quest towards a Mathematical Theory of Living Systems, pp. 85-117, Springer, Berlin, Germany, 2017.

[6] W. Yin, G. Fu, C. Yang, Z. Jiang, K. Zhu, and Y. Gao, "Fatal gas explosion accidents on Chinese coal mines and the characteristics of unsafe behaviors: 2000-2014," Safety Science, vol. 92, pp. 173-179, 2017.

[7] F. Ghasemi, O. Kalatpour, A. Moghimbeigi, and I. Mohhamadfam, "A path analysis model for explaining unsafe behavior in workplaces: the effect of perceived work pressure," International Journal of Occupational Safety and Ergonomics, vol. 24, no. 2, pp. 303-310, 2018.

[8] L. Li, S. Tian, and Y. Chen, "Influential factors index system of coal miners' unsafe behaviors based on sem-anp," Journal of Xian University of Science \& Technology, vol. 37, no. 4, pp. 529-536, 2017.

[9] N. Ben Khalifa, R. El-Azouzi, Y. Hayel, and I. Mabrouki, "Evolutionary games in interacting communities," Dynamic Games \& Applications, vol. 7, no. 2, pp. 1-26, 2016.

[10] H. Jia, Q. Cao, S. Wang, and S. Zhang, "Analysis of big data in coal mine and pre-control of workers' unsafe behaviors," Journal of Shandong University of Science \& Technology, vol. 34, no. 2, pp. 14-18, 2015.
[11] X. Wang, R. Lu, H. Yu, and D. Li, "Stability of the evolutionary game system and control strategies of behavior instability in coal mine safety management," Complexity, vol. 2019, Article ID 6987427, 14 pages, 2019.

[12] Q. Wang, Q. Mei, S. Liu, Q. Zhou, and J. Zhang, "Demographic differences in safety proactivity behaviors and safety management in Chinese small-scale enterprises," Safety Science, vol. 120, pp. 179-184, 2019.

[13] A. Prashar and M. Vijaya Sunder, "A bibliometric and content analysis of sustainable development in small and mediumsized enterprises," Journal of Cleaner Production, vol. 245, Article ID 118665, 2019.

[14] R. Tong, Y. Yang, X. Ma, Y. Zhang, S. Li, and H. Yang, "Risk assessment of miners' unsafe behaviors: a case study of gas explosion accidents in coal mine, China," International Journal of Environmental Research and Public Health, vol. 16, no. 10, p. $1765,2019$.

[15] B. S. Vadivoo, R. Ramachandran, J. Cao, H. Zhang, and X. Li, "Controllability analysis of nonlinear neutral-type fractionalorder differential systems with state delay and impulsive effects," International Journal of Control, Automation and Systems, vol. 16, no. 2, pp. 659-669, 2018.

[16] X. Li and J. Wu, "Stability of nonlinear differential systems with state-dependent delayed impulses," Automatica, vol. 64, pp. 63-69, 2016.

[17] M. Chi and W. Zhao, "Dynamical analysis of two-microorganism and single nutrient stochastic chemostat model with monod-haldane response function," Complexity, vol. 2019, Article ID 8719067, 13 pages, 2019.

[18] B. Hu, Z.-H. Guan, G. Chen, and F. L. Lewis, "Multistability of delayed hybrid impulsive neural networks with application to associative memories," IEEE Transactions on Neural Networks and Learning Systems, vol. 30, no. 5, pp. 1537-1551, 2019.

[19] T. Zhang, W. Ma, and X. Meng, "Global dynamics of a delayed chemostat model with harvest by impulsive flocculant input," Advances in Difference Equations, vol. 2017, no. 1, p. 115, 2017.

[20] J. Liu, Z. Zhao, W. Yu, and T. Zhang, "Triple solutions for a damped impulsive differential equation," Advances in Difference Equations, vol. 2019, no. 1, pp. 1-8, 2019.

[21] A. Zada and S. O. Shah, "Hyers-ulam stability of first-order non-linear delay differential equations with fractional integrable impulses," Hacettepe Journal of Mathematics and Statistics, vol. 47, no. 6, pp. 1196-1205, 2017.

[22] Q. Sun, H. Ji, and Y. Cui, "Positive solutions for boundary value problems of fractional differential equation with integral boundary conditions," Journal of Function Spaces, vol. 2018, Article ID 6461930, 6 pages, 2018.

[23] M. Chi and W. Zhao, "Dynamical analysis of multi-nutrient and single microorganism chemostat model in a polluted environment," Advances in Difference Equations, vol. 2018, no. 1, p. 120, 2018.

[24] W. Zhao, J. Liu, M. Chi, and F. Bian, "Dynamics analysis of stochastic epidemic models with standard incidence," Advances in Difference Equations, vol. 2019, no. 1, p. 22, 2019.

[25] L. Li, C. Li, and W. Zhang, "Existence of solution, pulse phenomena and stability criteria for state-dependent impulsive differential equations with saturation," Communications in Nonlinear Science and Numerical Simulation, vol. 77, pp. 312-323, 2019.

[26] X. Li, H. Li, and B. Wu, "Piecewise reproducing kernel method for linear impulsive delay differential equations with piecewise constant arguments," Applied Mathematics and Computation, vol. 349, pp. 304-313, 2019. 
[27] J. Liu and W. Zhao, "Dynamic analysis of stochastic lotka-volterra predator-prey model with discrete delays and feedback control," Complexity, vol. 2019, Article ID 4873290, 15 pages, 2019.

[28] C. Wang, J. Wang, X. Wang, H. Yu, L. Bai, and Q. Sun, "Exploring the impacts of factors contributing to unsafe behavior of coal miners," Safety Science, vol. 115, pp. 339-348, 2019.

[29] R. Lu, X. Wang, H. Yu, and D. Li, "Multiparty evolutionary game model in coal mine safety management and its application," Complexity, vol. 2018, Article ID 9620142, 10 pages, 2018.

[30] L. Wang, Q. Cao, and L. Zhou, "Research on the influencing factors in coal mine production safety based on the combination of dematel and ism," Safety Science, vol. 103, pp. 51-61, 2018.

[31] J. L. Stratman and C. M. Youssef-Morgan, "Can positivity promote safety? psychological capital development combats cynicism and unsafe behavior," Safety Science, vol. 116, pp. 13-25, 2019.

[32] S. Guo, P. Zhang, and L. Ding, "Time-statistical laws of workers' unsafe behavior in the construction industry: a case study," Physica A: Statistical Mechanics and Its Applications, vol. 515, pp. 419-429, 2019.

[33] K. Yu, Q. Cao, and L. Zhou, "Study on qualitative simulation technology of group safety behaviors and the related software platform," Computers \& Industrial Engineering, vol. 127, pp. 1037-1055, 2019.

[34] R. Lu, X. Wang, and D. Li, "A fractional supervision game model of multiple stakeholders and numerical simulation," Mathematical Problems in Engineering, vol. 2017, Article ID 9123624, 9 pages, 2017.

[35] H. Chen, Q. Feng, D. Zhu, S. Han, and R. Long, "Impact of rent-seeking on productivity in Chinese coal mine safety supervision: a simulation study," Energy Policy, vol. 93, pp. 315-329, 2016.

[36] Y.-Q. Wang, Y. Teng, J.-L. Wang, and L.-L. Zhang, "Research on decision-making behavior evolution of government, coal mine enterprises and employees under safe education," Eurasia Journal of Mathematics, Science and Technology Education, vol. 13, no. 12, pp. 8267-8281, 2017.

[37] M. Liu and S. Yang, "Research on the construction of coal legal system in the past 40 years of the reform and openingup," American Journal of Environmental and Resource Economics, vol. 4, no. 3, pp. 92-95, 2019.

[38] B. Xin, L. Liu, G. Hou, and Y. Ma, "Chaos synchronization of nonlinear fractional discrete dynamical systems via linear control," Entropy, vol. 19, no. 7, p. 351, 2017.

[39] B. Xin, W. Peng, Y. Kwon, and Y. Liu, "Modeling, discretization, and hyperchaos detection of conformable derivative approach to a financial system with market confidence and ethics risk," Advances in Difference Equations, vol. 2019, no. 1, p. 138, 2019.

[40] D. Aimene, D. Baleanu, and D. Seba, "Controllability of semilinear impulsive atangana-baleanu fractional differential equations with delay," Chaos, Solitons \& Fractals, vol. 128, pp. 51-57, 2019. 